\title{
Strong practical stability and stabilization of discrete linear repetitive processes
}

\author{
Paweł Dąbkowski • Krzysztof Gałkowski • \\ Eric Rogers • Anton Kummert
}

Received: 28 April 2008 / Revised: 26 September 2008 / Accepted: 3 February 2009 /

Published online: 13 March 2009

(C) Springer Science+Business Media, LLC 2009

\begin{abstract}
This paper considers two-dimensional (2D) discrete linear systems recursive over the upper right quadrant described by well known state-space models. Included are discrete linear repetitive processes that evolve over subset of this quadrant. A stability theory exists for these processes based on a bounded-input bounded-output approach and there has also been work on the design of stabilizing control laws, elements of which have led to the assertion that this stability theory is too strong in many cases of applications interest. This paper develops so-called strong practical stability as an alternative in such cases. The analysis includes computationally efficient tests that lead directly to the design of stabilizing control laws, including the case when there is uncertainty associated with the process model. The results are illustrated by application to a linear model approximation of the dynamics of a metal rolling process.
\end{abstract}

Keywords 2D information propagation $\cdot$ Recursive updating $\cdot$ Stability $\cdot$ Stabilization

\section{Introduction}

The systems theoretic properties of discrete linear systems recursive in the upper right quadrant of the $2 \mathrm{D}$ plane have been the subject of much investigation over the years using, in the

\footnotetext{
P. Dąbkowski $(\bowtie) \cdot$ K. Gałkowski

Institute of Physics, Nicolaus Copernicus University in Torun, Torun, Poland

e-mail: p.dabkowski@fizyka.umk.pl

K. Gałkowski

e-mail: k.galkowski@issi.uz.zgora.pl

E. Rogers

School of Electronics and Computer Science, University of Southampton, Southampton, UK

e-mail: etar@ecs.soton.ac.uk
}

A. Kummert

Faculty of Electrical, Information and Media Engineering, University of Wuppertal, Wuppertal, Germany

e-mail: kummert@uni-wuppertal.de 
main, the well known state-space models due to Roesser (1975) and Fornasini and Marchesini (1978) respectively. In particular, asymptotic or bounded-input bounded-output (BIBO) stability has been formulated and many tests for this property now exist. Also there has been substantial progress on other aspects of the systems theory for them.

Discrete linear repetitive processes are also recursive in the upper right quadrant of the $2 \mathrm{D}$ plane but here information in one of the two directions of information propagation is limited to a finite duration. In particular, the unique characteristic of such a process is a series of sweeps, termed passes, through a set of dynamics defined over a fixed finite duration known as the pass length. On each pass an output, termed the pass profile, is produced which acts as a forcing function on, and hence contributes to, the dynamics of the next pass profile. This, in turn, leads to the unique feature in that the output sequence of pass profiles generated can contain oscillations that increase in amplitude in the pass-to-pass direction.

Physical examples of these processes in the process industries are detailed in Rogers et al. (2007). Also in recent years applications have arisen where adopting a repetitive process setting for analysis has distinct advantages over alternatives. Examples of these so-called algorithmic applications include classes of iterative learning schemes in control/signal processing (Amann et al. 1998) and iterative algorithms for solving nonlinear dynamic optimization problems based on the maximum principle (Roberts 2002). In this last case, for example, use of the repetitive process setting provides the basis for the development of highly reliable and efficient iterative solution algorithms.

Repetitive process analysis starts from a stability theory (Rogers et al. 2007) which is of the BIBO form, i.e. bounded inputs are required to produce bounded sequences (i.e. process outputs) of pass profiles and (in its strongest form) is termed stability along the pass. Also it is possible to write the dynamics of a large sub-class of discrete linear repetitive processes in Roesser (or Fornasini-Marchesini) state-space model form and it has been shown that stability along the pass of a given example is equivalent to asymptotic or BIBO stability of its 2D Roesser (or Fornasini-Marchesini) model interpretation (Boland and Owens 1980).

In the $2 \mathrm{D}$ (and more generally $n \mathrm{D}, n \geq 3$ ) discrete linear systems case there has been work which has argued that asymptotic or BIBO stability is too strong in many cases of practical interest. This raised the question of whether or not there is another useful definition of this property and led to so-called practical BIBO stability for $n \mathrm{D}$ linear systems-see, for example, Agathoklis and Bruton (1983); Xu et al. (1994, 1997). The stability equivalence (Boland and Owens 1980) means that the practical stability analysis can also be applied to discrete linear repetitive processes (obviously the same sub-class for which this equivalence holds).

Here we first demonstrate that practical stability can be too weak when applied to discrete linear repetitive processes and then proceed to develop a stronger concept termed strong practical stability. We also show how this can be characterized by easily computed tests that lead on to control law design algorithms, including the case when there is uncertainty associated with the process model. Finally, we give and illustrative example based on a metal rolling application.

Throughout this paper, the null and identity matrices with the required dimensions are denoted by 0 and $I$ respectively. Moreover, $M>0(<0)$ denotes a real symmetric positive (negative) definite matrix, $\operatorname{Sym}\{M\}$ is used to denote $M+M^{T}$, and $*$ is used to denote transposed block entries in the symmetric Linear Matrix Inequalities (LMIs) that are the means by which the necessary computations can be completed for a given numerical example. 


\section{Background}

The state-space model of a discrete linear repetitive process (Rogers et al. 2007) has the following form over $0 \leq p \leq \alpha-1, k \geq 0$,

$$
\begin{aligned}
x_{k+1}(p+1) & =A x_{k+1}(p)+B u_{k+1}(p)+B y_{0}(p) \\
y_{k+1}(p) & =C x_{k+1}(p)+D u_{k+1}(p)+D y_{k}(p)
\end{aligned}
$$

where $\alpha<\infty$ is the pass length and on pass $k x_{k}(p) \in \mathbb{R}^{n}$ is the state vector, $y_{k}(p) \in \mathbb{R}^{m}$ is the pass profile vector, and $u_{k}(p) \in \mathbb{R}^{r}$ is the vector of control inputs. The boundary conditions are the pass state initial vector sequence and the initial pass profile and here these are assumed to be of the form

$$
x_{k+1}(0)=d_{k+1}, \quad k \geq 0, \quad y_{0}(p)=f(p), \quad 0 \leq p \leq \alpha-1
$$

where the $n \times 1$ vector $d_{k+1}$ has known constant entries and $f(p)$ is an $m \times 1$ vector whose entries are known functions of $p$.

The updating structure of both the state and pass profile (output) dynamics exhibits similarities with the well known Roesser (1975) and Fornasini and Marchesini (1978) state-space models for $2 \mathrm{D}$ quarter plane causal discrete linear systems and it is possible to solve some systems theoretic problems by exploiting such links. In essence, the route for stability analysis is to treat $\Phi=\left[\begin{array}{ll}A & B_{0} \\ C & D_{0}\end{array}\right]$ as the system matrix in a Roesser model interpretation of the repetitive process dynamics.

It is not true that all other systems theoretic problems for discrete linear repetitive processes can be solved by recourse to a Roesser or Fornasini-Marchesini model of the dynamics. For example, some applications require that the initial state vector on each pass is a function of points along the previous pass, and it is possible that these alone can cause instability. Such conditions have no Roesser or Fornasini-Marchesini model counterparts. Also there is no equivalent of the concept of pass profile controllability, i.e. the existence of a control input sequence which will force the process to produce a pre-specified state or pass profile vector on a specified pass. See Rogers et al. (2007) and the relevant cited references for a detailed treatment of systems theoretic problems which are unique to repetitive processes.

The stability theory (Rogers et al. 2007) for linear repetitive processes is based on an abstract model in a Banach space setting which includes a wide range of such processes as special cases, including those described by (1) and (2). In terms of their dynamics it is the pass-to-pass coupling (noting again their unique feature) which is critical. This is of the form $y_{k+1}=L_{\alpha} y_{k}$, where $y_{k} \in E_{\alpha}\left(E_{\alpha}\right.$ a Banach space with norm $\left.\|\cdot\|\right)$ and $L_{\alpha}$ is a bounded linear operator mapping $E_{\alpha}$ into itself. (In the case considered here $L_{\alpha}$ is a discrete convolution operator.)

Asymptotic stability, i.e. BIBO stability over the fixed finite pass length $\alpha>0$, requires the existence of finite real scalars $M_{\alpha}>0$ and $\lambda_{\alpha} \in(0,1)$ such that $\left\|L_{\alpha}^{k}\right\| \leq M_{\alpha} \lambda_{\alpha}^{k}, k \geq 0$ (where $\|\cdot\|$ also denotes the induced operator norm). For processes described by (1) and (2) this condition holds if, and only if, all eigenvalues of the matrix $D_{0}$ have modulus strictly less than unity, written here as $r\left(D_{0}\right)<1$ where $r(\cdot)$ denotes the spectral radius of its matrix argument.

Suppose that this condition holds and the input sequence applied $\left\{u_{k+1}\right\}_{k \geq 0}$ converges strongly as $k \rightarrow \infty$ (i.e. in the sense of the norm on the underlying function space) to $u_{\infty}$. Then the strong limit $y_{\infty}:=\lim _{k \rightarrow \infty} y_{k}$ is termed the limit profile corresponding to this input sequence and its dynamics along the pass are described by 


$$
\begin{aligned}
x_{\infty}(p+1) & =\left(A+B_{0}\left(I-D_{0}\right)^{-1} C\right) x_{\infty}(p)+\left(B+B_{0}\left(I-D_{0}\right)^{-1} D\right) u_{\infty}(p) \\
y_{\infty}(p) & =\left(I-D_{0}\right)^{-1} C x_{\infty}(p)+\left(I-D_{0}\right)^{-1} D u_{\infty}(p) \\
x_{\infty}(0) & =d_{\infty}
\end{aligned}
$$

where $d_{\infty}$ is the strong limit of the sequence $\left\{d_{k}\right\}_{k \geq 1}$. In physical terms, this result states that under asymptotic stability the repetitive dynamics can, after a 'sufficiently large' number of passes have elapsed, be replaced by those of a 1D discrete linear system. In particular, this property demands that the amplifying properties of the coupling between successive pass profiles are completely damped out (in the $k$ direction) after a sufficiently large number of passes have elapsed.

To provide another interpretation of asymptotic stability, consider the case when $p=0$ with $d_{k+1}=0, k \geq 1$ and zero input signal on each pass. Then $y_{k}(0)=D_{0}^{k} y_{0}(0)$ and hence the process will be asymptotically stable provided the sequence of initial pass profile vectors does not become unbounded. In particular, asymptotic stability is independent of the state updating dynamics and this could be a source of major difficulty in at least some cases.

Note 1 The limit profile as $k \rightarrow \infty$ is termed horizontal to distinguish it from the case introduced below when $p \rightarrow \infty$ that will be termed the vertical limit profile.

Although, we demand for the definition of the horizontal limit profile only a finite and fixed pass length, it is straightforward to see from the state updating equation of (3) that it can be treated as the 1D discrete linear system in the $p$ direction. Consider also the case when $A=-0.5, B=1, B_{0}=0.5+\beta, \beta$ a real scalar, $C=1$ and $D=D_{0}=0$. This is an asymptotically stable process with resulting limit profile (with zero state initial vector sequence) $y_{\infty}(p+1)=\beta y_{\infty}(p)+u_{\infty}(p)$, which is unstable as a 1D discrete linear system if $|\beta| \geq 1$, i.e. the dynamic response increases in magnitude as $p$ (the long the pass variable) evolves over the finite interval $[0, \alpha]$. In many cases, such a situation will not be acceptable, e.g. in a control application where the task is to force the process to track a given target or reference vector. For other cases, however, asymptotic stability will suffice or indeed is all that can ever be achieved (see the application in Roberts 2002).

If a horizontal limit profile which is unstable in the 1D linear systems sense is not acceptable then we demand the BIBO property for all possible values of the pass length. Mathematically, this can be analyzed by letting $\alpha \rightarrow \infty$. This is stability along the pass, see Rogers et al. (2007). Recall also that the repetitive processes here can be interpreted in 2D Roesser or Fornasini-Marchesini state-space model terms where for stability analysis the route is via the matrix $\Phi$ defined earlier in this section. Then it can be shown that stability along the pass of the repetitive processes considered here is equivalent to asymptotic stability of its 2D discrete linear systems interpretation (Boland and Owens 1980; Rogers et al. 2007). This stability equivalence does not hold for any other sub-classes of repetitive processes.

Stability along the pass must hold for $k \rightarrow \infty$ and $p \rightarrow \infty$ simultaneously and several sets of necessary and sufficient conditions (Rogers et al. 2007) for this property are known, such as the following.

Theorem 1 Suppose that the pair $\left\{A, B_{0}\right\}$ is controllable and the pair $\{C, A\}$ is observable. Then a discrete linear repetitive process described by (1) and (2) is stable along the pass if, and only if, $r\left(D_{0}\right)<1, \quad r(A)<1$ and all eigenvalues of

$$
G(z)=C(z I-A)^{-1} B_{0}+D_{0}
$$

have modulus strictly less than unity $\forall|z|=1$ 
These conditions can, in principle, be tested by direct application of well known 1D discrete linear systems tests. Application of them to the example given above shows that stability along the pass also places a constraint on the state dynamics on both the current pass $(r(A)<1)$ and (in the single-input single-output case for simplicity) the complete frequency response of the transfer-function describing the contribution of the previous pass profile, and not just on the matrix $D_{0}\left(=\lim _{|z| \rightarrow \infty} G(z)\right)$ (in particular, a constraint on the coupling between the previous pass profile and current pass state dynamics through the $B_{0}$ matrix). Also it is easy to see that stability along the pass ensures that the resulting limit profile is stable as a $1 \mathrm{D}$ discrete linear system, i.e. $r\left(A+B_{0}\left(I-D_{0}\right)^{-1} C\right)<1$ (or a limit profile which is stable as a $1 \mathrm{D}$ linear system is a necessary condition for stability along the pass).

The only difficulty with using this last result is that testing (4) could be computationally intensive and, despite its Nyquist basis, it has not proved to be a starting point for onward analysis, e.g. the design of a stabilizing control law, except in a few very restrictive special cases. This is in contrast to the Nyquist stability test for 1D linear systems. The most effective way currently available for control systems/filter design is via LMIs, e.g. Galkowski et al. (2002). This results in stability tests which can be implemented by computations with matrices which have constant entries and also a straightforward extension is possible to, for example, enable the design of control laws for stability along the pass and/or filter design (Wu et al. 2009).

The price paid for this progress is that the LMI based analysis works on sufficient, as opposed to necessary and sufficient conditions (such as those of Theorem 1) and hence there will be a degree of conservativeness associated with all results and analysis that start from this basis. There is hence great potential benefit in seeking an alternative approach that does not have this undesirable feature. It is, of course, possible to work with asymptotic stability alone but as we have already argued above it is much too weak in many cases.

As already noted in this paper, another way to consider stability of 2D systems, and hence discrete linear repetitive processes of the form considered here, is to use the weaker concept of practical stability (Agathoklis and Bruton 1983; Xu et al. 1994, 1997). Applying the resulting conditions to the $2 \mathrm{D}$ linear systems interpretation of processes described by (1) and (2) gives the following result.

Lemma 1 A discrete linear repetitive process described by (1) is practically stable if, and only if, $r\left(D_{0}\right)<1$ and $r(A)<1$.

In terms of applying this result to a given example, we simply need to complete two tests for the $1 \mathrm{D}$ discrete linear systems property.

Consider now an industrial example such as a gantry robot whose task is to collect an object from a location and place it on a moving conveyor belt after a finite time has elapsed, then return to the original location to pick up the next one and so on. This is an obvious application for iterative learning control (Ratcliffe et al. 2006), and hence repetitive process theory, in that the time taken to complete the return journey can be used to update the control law using previous pass information to sequentially improve performance. Maximum benefit will arise here if this operation can be executed a very large number of times without the need to stop and hence lose throughput. Hence we have the case when $\alpha$ is finite and $k \rightarrow \infty$ and for this we clearly need a form of stability where the horizontal limit profile, i.e. $k \rightarrow \infty$, exists with stable along the pass dynamics and, ideally, acceptable tracking of the reference signal can be achieved. We already know that this requires asymptotic stability plus a stable limit profile where the latter requirement cannot be guaranteed by practical stability. (The conditions of Lemma 1 do not guarantee a limit profile that is stable in the $p$ direction as the 
example given earlier in this paper, i.e. $A=-0.5, B=1, B_{0}=0.5+\beta, \beta$ a real scalar, $C=1$ and $D=D_{0}=0$ ) demonstrates).

In the remainder of this paper we develop strong practical stability as an alternative to the property of Lemma 1 by removing the uniform boundedness requirement as both $k \rightarrow \infty$ and $\alpha \rightarrow \infty$ but still demanding this property when (i) both $k$ and $\alpha$ are finite, (ii) the pass index $k \rightarrow \infty$ and the pass length $\alpha$ finite, and (iii) the pass index $k$ is finite and the pass length $\alpha \rightarrow \infty$. Cases (i) and (ii) are already practically motivated and Case (iii) is the mathematical formulation where the process completes a finite number of passes but the pass length is 'very long' and there is a requirement to control the along the pass dynamics. We also develop LMI based tests for the resulting necessary and sufficient conditions that lead directly to control law design algorithms, including the case when there is uncertainty associated with the process model.

\section{Strong practical stability}

Consider the case of $p=0$ with zero state initial vector sequence and zero control input vector. Then $y_{k}(0)=D_{0}^{k} y_{0}(0)$ and hence we require $r\left(D_{0}\right)<1$. Under this condition, i.e. asymptotic stability, we achieve the limit profile (3) as $k \rightarrow \infty$ that is stable when $r\left(A+B_{0}\left(I-D_{0}\right)^{-1} C\right)<1$.

Consider now any finite $k$. Then clearly (consider the case when there is no previous pass profile contribution) we require that $r(A)<1$. Also, again with zero state initial vector sequence and zero control input vector, as $p \rightarrow \infty$

$$
y_{k+1}(\infty)=\left(C(I-A)^{-1} B_{0}+D_{0}\right) y_{k}(\infty)
$$

and hence we require $r\left(C(I-A)^{-1} B_{0}+D_{0}\right)<1$. In summary, therefore, strong practical stability requires that the following conditions hold

[a] $r\left(D_{0}\right)<1$

[b] $r(A)<1$

[c] $r\left(A+B_{0}\left(I-D_{0}\right)^{-1} C\right)<1$, and

[d] $r\left(C(I-A)^{-1} B_{0}+D_{0}\right)<1$

The conditions for strong practical stability can, assuming no numerical problems with computing the eigenvalues of the matrices involved, be easily checked for a given example. Suppose, however, that the task is to ensure this property by application of a control law (see also the next section) of the form

$$
u_{k+1}(p)=K_{1} x_{k+1}(p)+K_{2} y_{k}(p)
$$

which is a combination of current pass state feedback plus a feedforward term from the previous pass profile (in keeping with the fact that use of only current pass state or pass profile vector activated control laws cannot stabilize the process dynamics in all but a few restrictive special cases). Then when this control law is applied the process state-space model matrices $A, B_{0}, C, D_{0}$ are mapped to $A+B K_{1}, B_{0}+F K_{2}, C+D K_{1}$, and $D_{0}+D K_{2}$ respectively. Hence design to satisfy conditions [a] and [b] for the controlled process is simply two applications of the 1D pole placement problem for discrete linear systems. The case for conditions [c] and $[\mathrm{d}]$ is far from clear and hence as a preliminary step to overall control law design we make novel use of results from 1D singular discrete linear systems theory for the state-space model

$$
E x(h+1)=\widehat{A} x(h)+\widehat{B} u(h)
$$


where $E$ is a singular matrix. In particular, condition [c] is easily seen to be equivalent to stability of the $1 \mathrm{D}$ singular linear system with state-space model

$$
E_{1} z(h+1)=A_{1} z(h)+\Pi u(h)
$$

where $E_{1}=\left[\begin{array}{ll}I & 0 \\ 0 & 0\end{array}\right], A_{1}=\left[\begin{array}{cc}A & B_{0} \\ C & D_{0}-I\end{array}\right], \Pi=\left[\begin{array}{c}B \\ D\end{array}\right]$. Similarly, condition $[\mathrm{d}]$ is equivalent to stability of the $1 \mathrm{D}$ singular linear system

$$
E_{2} z(h+1)=A_{2} z(h)+\Pi u(h)
$$

where $E_{2}=\left[\begin{array}{ll}0 & 0 \\ 0 & I\end{array}\right], A_{2}=\left[\begin{array}{cc}A-I & B_{0} \\ C & D_{0}\end{array}\right]$

We also need the following definition.

Definition 1 A 1D discrete singular linear system of the form (7) is termed admissible (Dai 1989) if it is stable, regular, i.e. $\operatorname{det}(z E-\widehat{A})$ is not identically zero, and $\operatorname{deg}(\operatorname{det}(z E-\widehat{A}))=$ $\operatorname{rank}(E)$.

It is clear that $\exists$ (with compatible dimensions) nonsingular matrices $U$ and $V$ such that

$$
U E V=\left[\begin{array}{ll}
I & 0 \\
0 & 0
\end{array}\right]
$$

Also introduce $E^{\perp}=V(I-U E V) U$ and $E^{\ddagger}=U^{T}(I-U E V) U^{-T}$. Then we have the following result (Chaabane et al. 2007).

Lemma 2 A $1 D$ discrete singular linear system of the form (7) is admissible if, and only if, $\exists$ matrices $X, Y$ and $G$ (with compatible dimensions) such that the following LMI is feasible for a given $\beta>1$

$$
\left[\begin{array}{cc}
-E X E^{T} & \left(E^{\perp} Y E^{\ddagger}\right)^{T} \\
E^{\perp} Y E^{\ddagger} & X
\end{array}\right]+\operatorname{Sym}\left\{\left[\begin{array}{c}
\widehat{A} G \\
-G
\end{array}\right]\left[\begin{array}{ll}
U^{-T} \beta V^{T}
\end{array}\right]\right\}<0
$$

Note here that, with the aim of extending the analysis to stability and control law design for uncertain processes (see Sect. 5), additional slack matrices have been introduced in the above Lemma as in de Oliveira et al. (1999) or Peaucelle et al. (2000). This should lower the level of conservativeness and assist in avoiding ill-conditioning of intermediate results that can strongly affect the final outcome in numerical computations.

Now we can give the first new result of this paper in the form of computable necessary and sufficient conditions for strong practical stability. (This result is subsequently used as a basis for control law design, including the case when there is uncertainty associated with the process model.)

Lemma 3 A discrete linear repetitive process described by (1) and (2) is strongly practically stable if, and only if, $\exists$ matrices $W_{1}>0, W_{2}>0, X_{21}^{1}, X_{21}^{2}, X_{11}^{1}=\left(X_{11}^{1}\right)^{T}, X_{22}^{1}=\left(X_{22}^{1}\right)^{T}$, $Y_{11}^{2}, Y_{22}^{1}, X_{11}^{2}=\left(X_{11}^{2}\right)^{T}, X_{22}^{2}=\left(X_{22}^{2}\right)^{T}, \widetilde{G_{1}}, \widetilde{G_{2}}$ (of compatible positions) such that the following LMIs are feasible for scalars $\beta_{1}>1, \beta_{2}>1$ 


$$
\begin{aligned}
& \quad\left[\begin{array}{cc}
-W_{1} & W_{1}^{T} D_{0}^{T} \\
D_{0} W_{1} & -W_{1}
\end{array}\right]<0 \\
& {\left[\begin{array}{ccc}
-W_{2} & W_{2}^{T} A^{T} \\
A W_{2} & -W_{2}
\end{array}\right]<0} \\
& {\left[\begin{array}{cccc}
-X_{11}^{1} & 0 & 0 & 0 \\
0 & 0 & 0 & \left(Y_{22}^{1}\right)^{T} \\
0 & 0 & X_{11}^{1} & \left(X_{21}^{1}\right)^{T} \\
0 & Y_{22}^{1} & X_{21}^{1} & X_{22}^{1}
\end{array}\right]+\operatorname{Sym}\left\{\left[\begin{array}{c}
A_{1} \widetilde{G_{1}} \\
-\widetilde{G_{1}}
\end{array}\right]\left[\begin{array}{ll}
I & \beta_{1} I
\end{array}\right]\right\}<0} \\
& {\left[\begin{array}{cccc}
0 & 0 & \left(Y_{11}^{2}\right)^{T} & 0 \\
0 & -X_{22}^{2} & 0 & 0 \\
Y_{11}^{2} & 0 & X_{11}^{2} & \left(X_{21}^{2}\right)^{T} \\
0 & 0 & X_{21}^{2} & X_{22}^{2}
\end{array}\right]+\operatorname{Sym}\left\{\left[\begin{array}{c}
A_{2} \widetilde{G_{2}} \\
-\widetilde{G_{2}}
\end{array}\right]\left[\begin{array}{ll}
U_{2} & \beta_{2} U_{2}
\end{array}\right]\right\}<0}
\end{aligned}
$$

where $U_{2}:=\left[\begin{array}{ll}0 & I \\ I & 0\end{array}\right]$

Proof The LMIs of (12) and (13) are commonly used in 1D linear systems stability theory. Also interpreting the conditions of Lemma 2 in terms of (8) and (9) shows that LMIs (14) and (15) are equivalent to requirements [c] and [d] respectively for strong practical stability. (Note also that in Lemma 2 applied to both cases only the block entries $Y_{11}^{2}$ and $Y_{22}^{1}$ respectively in the $(n+m) \times(n+m)$ matrix $Y$ influence the final result).

To illustrate the differences between the various types of stability for discrete linear repetitive processes considered in this paper, we now give some simulation examples where in each case the pass profile and input vectors are scalars. Also all simulations have zero input signal applied and hence we are examining the response to non-zero boundary conditions-note again the critical role these have in the stability characteristics of these processes.

We begin with Fig. 1 that shows the pass profile sequence for an asymptotically unstable, i.e. $r\left(D_{0}\right) \geq 1$, example and hence neither strong practical stability or stability along the pass can hold.

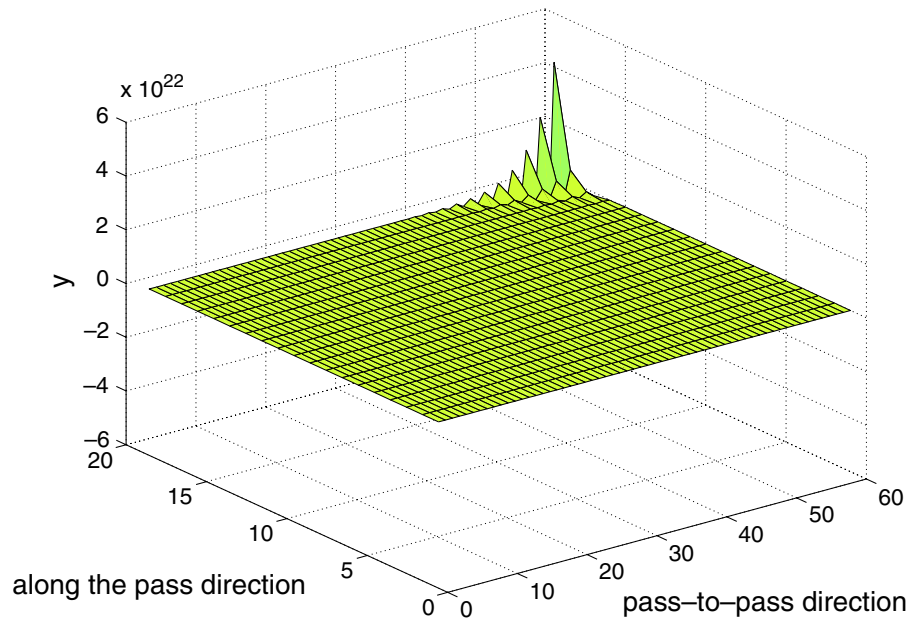

Fig. 1 Pass profile sequence for an asymptotically unstable process 


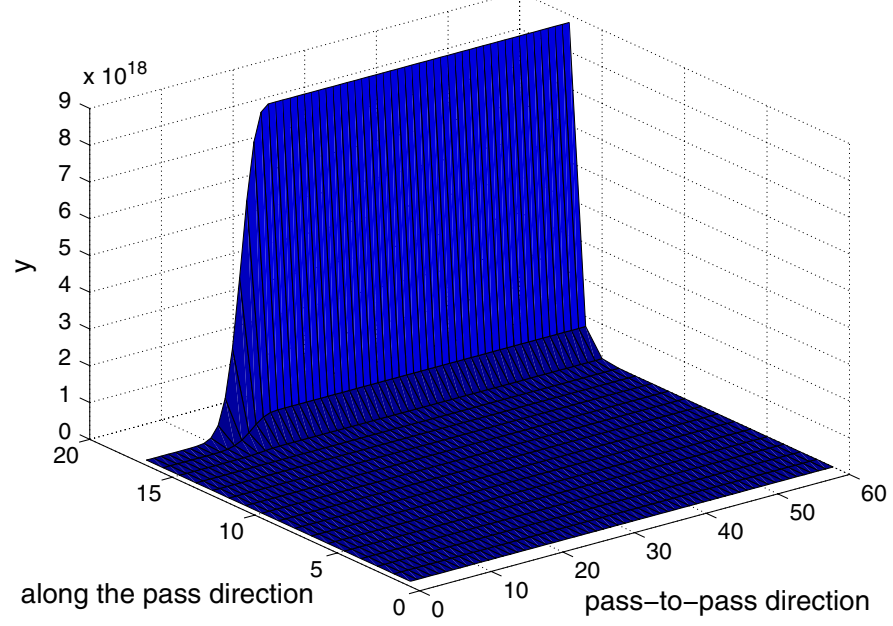

Fig. 2 Pass profile sequence for an asymptotically stable process

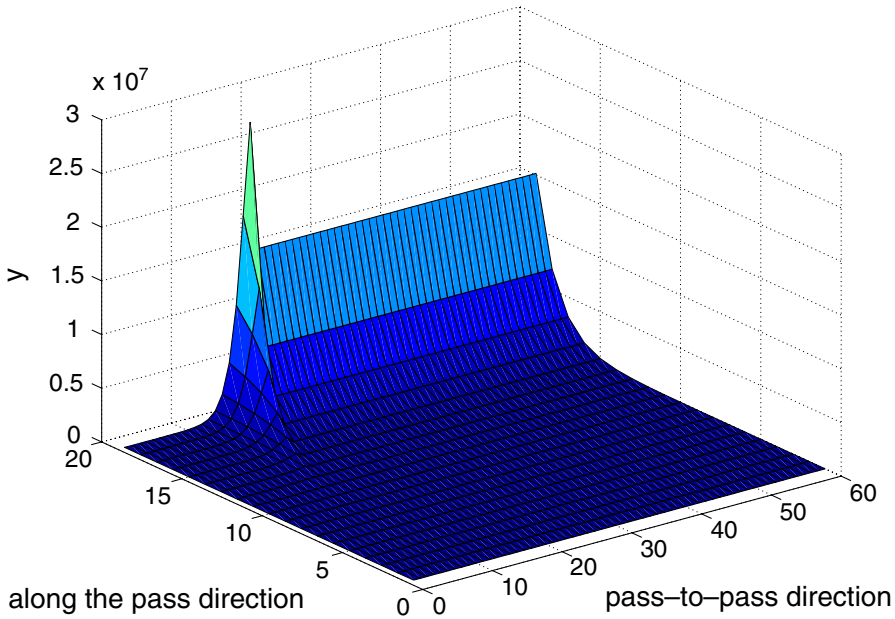

Fig. 3 Pass profile sequence of a practically stable process

Figure 2 shows the pass profile sequence for an example which is asymptotically stable, i.e. $r\left(D_{0}\right)<1$, (and hence a limit profile described by a 1D discrete linear system exists) but neither strong practical stability or stability along the pass hold. Here the pass profile sequence converges in the pass-to-pass $(k)$ direction but the resulting limit profile is unstable in the along the pass $(p)$ direction.

Figure 3 shows an example where practical stability, i.e. $r\left(D_{0}\right)<1$ and $r(A)<1$, holds and illustrates that under this property an unacceptable horizontal limit profile can result. In particular, such a limit profile is unacceptable in terms of applications where control action is applied to track a reference signal. This unacceptable behavior arises because practical stability takes no account of the previous pass contributions (through the state-space model matrices $B_{0}$ and $D_{0}$ ) to the current pass profile. 


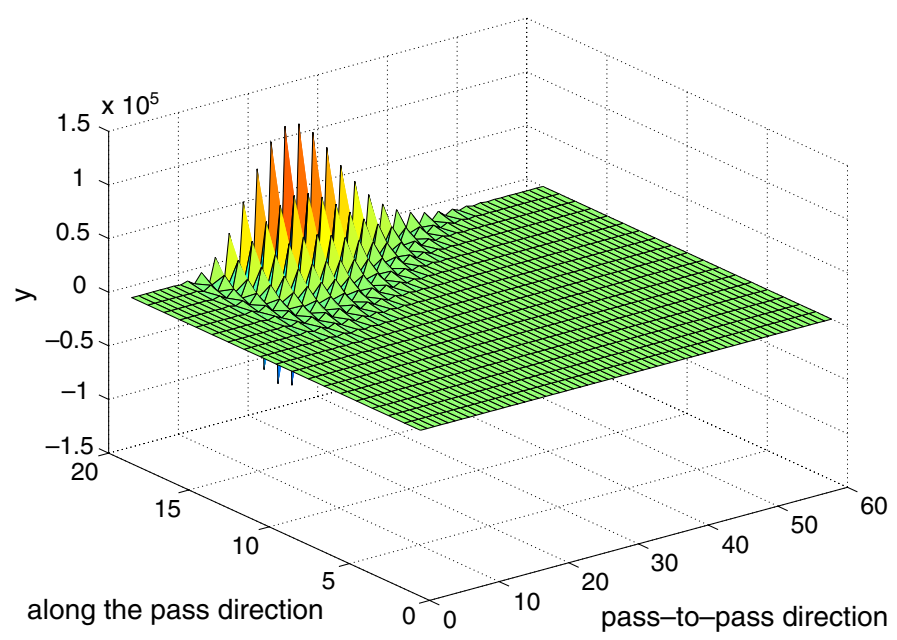

Fig. 4 Pass profile sequence of a strong practically stable process

Figure 4 shows an example that is strongly practically stable. The key point here is that the oscillations that arise are damped out as the process dynamics evolve in $k$. Clearly there will be cases when even this is unacceptable and will need either control action applied or stability along the pass with control action. For cases where strong practical stability (with control action if required) the core problem of determining if this property holds for a given example is much easier than the same task for stability along the pass (where in the latter case we must also check the frequency domain condition (4)).

It is also possible to characterize strong practical stability in terms of the poles of the example considered where it is first important to note that the concept of a pole for an $n \mathrm{D}$ linear system is much more complex than in the 1D case (Wood et al. 2000). For the discrete linear repetitive processes of the form considered here, however, the situation is less complex and can be used to explain the differences between strong practical stability and stability along the pass. The starting point is the characteristic polynomial for these processes defined as

$$
\rho\left(z_{1}, z_{2}\right)=\operatorname{det}\left(\left[\begin{array}{cc}
z_{1} I-A & -B_{0} \\
-C & z_{2} I_{m}-D_{0}
\end{array}\right]\right)
$$

and the poles are the component-wise non-zero points in 2D complex space where the matrix on the left-hand-side here fails to have full rank, i.e., they are given by

$$
\rho\left(z_{1}, z_{2}\right)=0
$$

and the set $\left\{a_{1}, a_{2}\right\}$ that satisfy this last equation is termed the pole-variety. Also stability along the pass holds if, and only if,

$$
\rho\left(z_{1}, z_{2}\right) \neq 0,\left|z_{1}\right| \geq 1,\left|z_{2}\right| \geq 1
$$

The poles here are given by the vanishing of a single 2D non-unit polynomial and it is guaranteed to be a $1 \mathrm{D}$ geometric set in $2 \mathrm{D}$ complex space. Note also that the pole variety must be complex, even though the entries of the matrices $A, B_{0}, C$ and $D_{0}$ are real. This is essential in order to capture the full exponential-type dynamics of the process. The poles of 
these processes can be interpreted in terms of exponential trajectories, which in which in the case considered here have a clear physical interpretation.

Assume that $\left(a_{1}, a_{2}\right) \in \mathbf{C}^{2}$ is such that $\rho\left(a_{1}, a_{2}\right)=0$, and write $a_{1}=r_{1} e^{\imath \theta_{1}}, a_{2}=r_{2} e^{\imath \theta_{2}}$ (with $r_{1}=0$ for $a_{1}=0$ and $r_{2}=0$ for $a_{2}=0$ ). Then the existence of such a pair guarantees (Wood et al. 2000, Theorem/Definition 4.4) the existence of an exponential trajectory in the process having the form

$$
\begin{aligned}
& x_{k}(p)=x_{00}^{1} r_{1}^{p} r_{2}^{k} \cos \left(\theta_{1} p+\theta_{2} k\right)+x_{00}^{2} r_{1}^{p} r_{2}^{k} \sin \left(\theta_{1} p+\theta_{2} k\right) \\
& y_{k}(p)=y_{00}^{1} r_{1}^{p} r_{2}^{k} \cos \left(\theta_{1} p+\theta_{2} k\right)+y_{00}^{2} r_{1}^{p} r_{2}^{k} \sin \left(\theta_{1} p+\theta_{2} k\right) \\
& u_{k}(p)=0
\end{aligned}
$$

From this we conclude that stability along the pass requires no poles with $\left|a_{1}\right| \geq 1,\left|a_{2}\right| \geq 1$, which is a direct generalization of the $1 \mathrm{D}$ linear systems case. For strong practical stability, it is easy to see that condition [c] is equivalent to $\rho\left(z_{1}, 1\right) \neq 0,\left|z_{1}\right| \geq 1$, and condition [d] to $\rho\left(1, z_{2}\right) \neq 0,\left|z_{2}\right| \geq 1$. Hence for this stability property the only exponential trajectories considered are identical to those for a 1D discrete linear system, and these are clearly a subset of those given in (19).

\section{Guaranteeing strong practical stability}

If the strong practical stability property is not present for a given example then in control and signal processing applications it will clearly be necessary to introduce regulation action to guarantee it. One way of doing this is to use the control law (6), i.e. $u_{k+1}(p)=$ $K_{1} x_{k+1}(p)+K_{2} y_{k}(p)$, which is the sum of current pass state feedback and a 'feedforward' term from the previous pass. A simpler structure would result if current pass state feedback alone could be used but it is known that this is only possible in a few very restrictive special cases. Note also that the previous pass profile is a measured output and here we assume that it not significantly corrupted by noise etc. Moreover, the current pass state vector in this control law could be replaced by the current pass profile or estimated using an observer if not all entries are available for measurement.

Application of (6) now gives the controlled process state-space model

$$
\begin{aligned}
x_{k+1}(p+1) & =\left(A+B K_{1}\right) x_{k+1}(p)+\left(B_{0}+B K_{2}\right) y_{k}(p) \\
y_{k+1}(p) & =\left(C+D K_{1}\right) x_{k+1}(p)+\left(D_{0}+D K_{2}\right) y_{k}(p)
\end{aligned}
$$

To achieve asymptotic stability for the controlled process, only $K_{2}$ needs to be selected by solving the 1D discrete linear systems problem with state matrix $D_{0}$, input matrix $D$ and control law matrix $K_{2}$. For practical stability, we must add the solution of the 1D discrete linear systems pole placement problem with state matrix $A$, input matrix $B$ and control law matrix $K_{1}$, i.e. solve two independent $1 \mathrm{D}$ discrete linear systems pole placement problems.

Despite its obvious appeal in terms of simplicity of the control law design problem, a controlled practically stable process may still exhibit the unacceptable response characteristics illustrated in Fig. 3 (for an uncontrolled process). In such cases, the next approach (before moving on to stability along the pass) is to design for strong practical stability and the following are necessary and sufficient conditions for this property (obtained by interpreting the conditions for strong practical stability in terms of this last state-space model). 
Theorem 2 A controlled discrete linear repetitive process described by (22) is strongly practically stable if, and only if,

$$
\begin{gathered}
r\left(D_{0}+D K_{2}\right)<1 \\
r\left(A+B K_{1}\right)<1 \\
r\left(\left(B_{0}+B K_{2}\right)\left(I-D_{0}-D K_{2}\right)^{-1}\left(C+D K_{1}\right)+\left(A+B K_{1}\right)\right)<1 \\
r\left(\left(C+D K_{1}\right)\left(I-A-B K_{1}\right)^{-1}\left(B_{0}+B K_{2}\right)+\left(D_{0}+D K_{2}\right)\right)<1
\end{gathered}
$$

The following result from 1D discrete singular linear systems theory is the basis for the remaining analysis in this paper (basically the analysis which yielded necessary and sufficient conditions for strong practical stability can be extended to allow control law design for this property).

Lemma 4 (Chaabane et al. 2007) The 1D discrete singular linear system resulting from applying the state feedback control law

$$
u(h)=K x(h)
$$

to (7) is admissible if, and only if, $\exists$ matrices $X, Y, G$, and $R$ such that the following $L M I$

$$
\left[\begin{array}{cc}
-E X E^{T} & \left(E^{\perp} Y E^{\ddagger}\right)^{T} \\
E^{\perp} Y E^{\ddagger} & X
\end{array}\right]+S y m\left\{\left[\begin{array}{c}
\widehat{A} G+\widehat{B} R \\
-G
\end{array}\right]\left[\begin{array}{ll}
U^{-T} & \beta V^{T}
\end{array}\right]\right\}<0
$$

is feasible for a given $\beta>1$. Also

$$
K=R G^{-1}
$$

results in a stable system.

Now we have the following result which gives a method for control law design to ensure strong practical stability.

Theorem 3 A controlled discrete linear repetitive process described by (22) is strongly practically stable if the following LMIs

$$
\begin{aligned}
& {\left[\begin{array}{cc}
W_{2}-G_{2}-G_{2}^{T} & * \\
D_{0} G_{2}+D R_{2} & -W_{2}
\end{array}\right]<0 } \\
& {\left[\begin{array}{cc}
W_{1}-G_{1}-G_{1}^{T} & * \\
A G_{1}+B R_{1} & -W_{1}
\end{array}\right]<0 } \\
& {\left[\begin{array}{cccc}
-X_{11}^{1} & 0 & 0 & 0 \\
0 & 0 & 0 & \left(Y_{22}^{1}\right)^{T} \\
0 & 0 & X_{11}^{1}\left(X_{21}^{1}\right)^{T} \\
0 & Y_{22}^{1} X_{21}^{1} & X_{22}^{1}
\end{array}\right]+S y m\left\{\begin{array}{cc}
A G_{1}+B R_{1} & B_{0} G_{2}+B R_{2} \\
C G_{1}+D R_{1} & D_{0} G_{2}-G_{2}+D R_{2} \\
-G_{1} & 0 \\
0 & -G_{2}
\end{array}\right.}
\end{aligned}
$$




$$
\begin{aligned}
& \left.\begin{array}{cc}
A G_{1} \beta_{1}+B R_{1} \beta_{1} & B_{0} G_{2} \beta_{1}+B R_{2} \beta_{1} \\
C G_{1} \beta_{1}+D R_{1} \beta_{1} & D_{0} G_{2} \beta_{1}-G_{2} \beta_{1}+D R_{2} \beta_{1} \\
-G_{1} \beta_{1} & 0 \\
0 & -G_{2} \beta_{1}
\end{array}\right\}<0 \\
& {\left[\begin{array}{cccc}
0 & 0 & \left(Y_{11}^{2}\right)^{T} & 0 \\
0 & -X_{22}^{2} & 0 & 0 \\
Y_{11}^{2} & 0 & X_{11}^{2} & \left(X_{21}^{2}\right)^{T} \\
0 & 0 & X_{21}^{2} & X_{22}^{2}
\end{array}\right]+\operatorname{Sym}\left\{\begin{array}{cc}
A G_{1}-G_{1}+B R_{1} & B_{0} G_{2}+B R_{2} \\
C G_{1}+D R_{1} & D_{0} G_{2}+D R_{2} \\
-G_{1} & 0 \\
0 & -G_{2}
\end{array}\right.} \\
& A G_{1} \beta_{2}-G_{1} \beta_{2}+B R_{1} \beta_{2} B_{0} G_{2} \beta_{2}+B R_{2} \beta_{2} \\
& C G_{1} \beta_{2}+D R_{1} \beta_{2} \quad D_{0} G_{2} \beta_{2}+D R_{2} \beta_{2} \\
& -G_{1} \beta_{2} \\
& 0 \\
& \left.\begin{array}{c}
0 \\
-G_{2} \beta_{2}
\end{array}\right\}<0
\end{aligned}
$$

hold for given $\beta_{1}>1$ and $\beta_{2}>1$ and matrix variables $W_{1}>0, W_{2}>0, X_{21}^{1}, X_{21}^{2}$, $X_{11}^{1}=\left(X_{11}^{1}\right)^{T}, X_{22}^{1}=\left(X_{22}^{1}\right)^{T}, Y_{11}^{2}, Y_{22}^{1}, X_{11}^{2}=\left(X_{11}^{2}\right)^{T}, X_{22}^{2}=\left(X_{22}^{2}\right)^{T}, G_{1}, G_{2}, R_{1}, R_{2}$. When these conditions hold, then stabilizing control law matrices are given by

$$
K_{1}=R_{1} G_{1}^{-1}, \quad K_{2}=R_{2} G_{2}^{-1}
$$

Proof Conditions (30) and (31) ensure that $r\left(A+B K_{1}\right)<1$ and $r\left(D_{0}+D K_{2}\right)<1$ respectively hold. The proofs for the last two conditions follow from the same arguments in terms of Lemma 2 as those relating to the use of Lemma 1 in establishing the corresponding conditions for the uncontrolled case. For the first of these

$$
R=\left[\begin{array}{ll}
R_{1} & R_{2}
\end{array}\right], \quad G=\left[\begin{array}{cc}
G_{1} & 0 \\
0 & G_{2}
\end{array}\right]
$$

and for the second

$$
R=\left[\begin{array}{ll}
R_{2} & R_{1}
\end{array}\right], \quad G=\left[\begin{array}{cc}
0 & G_{1} \\
G_{2} & 0
\end{array}\right]
$$

Routine mathematical manipulations then yield the required LMIs and the proof is complete.

\section{Robust stability and stabilization}

Often an exact model of the process dynamics will not be available. Hence we must consider control law design in the presence of uncertainty. Here we show how the analysis of the previous section can be extended to this general problem area where, in common with other areas of linear systems theory, we assume that the uncertainty present has one of two general forms, i.e. norm bounded or polytopic.

Norm-bounded uncertainty In this case we assume that the uncertain process dynamics are described by a state-space model of the following form

$$
\begin{aligned}
x_{k+1}(p+1) & =(A+\Delta A) x_{k+1}(p)+(B+\Delta B) u_{k+1}(p)+\left(B_{0}+\Delta B_{0}\right) y_{k}(p) \\
y_{k+1}(p) & =(C+\Delta C) x_{k+1}(p)+(D+\Delta D) u_{k+1}(p)+\left(D_{0}+\Delta D_{0}\right) y_{k}(p)
\end{aligned}
$$


where the matrices $\Delta A, \Delta B, \Delta B_{0}, \Delta C, \Delta D$ and $\Delta D_{0}$ representing admissible uncertainties are assumed to satisfy

$$
\left[\begin{array}{ccc}
\Delta A & \Delta B_{0} & \Delta B \\
\Delta C & \Delta D_{0} & \Delta D
\end{array}\right]=\left[\begin{array}{l}
H_{1} \\
H_{2}
\end{array}\right] \mathcal{F}\left[\begin{array}{lll}
M_{1} & M_{2} & M_{3}
\end{array}\right]
$$

and $H$ and $M$ are known matrices with constant entries (and compatible dimensions) and the unknown matrix $\mathcal{F}$ satisfies

$$
\mathcal{F}^{T} \mathcal{F} \leq I
$$

We will also require the following well known result

Lemma 5 Suppose we are given known real matrices $H, M$ and an unknown matrix $\mathcal{F}$ which satisfies $\mathcal{F}^{T} \mathcal{F} \leq I$. Then, for any $\epsilon>0$,

$$
H \mathcal{F} M+M^{T} \mathcal{F}^{T} H^{T} \leq \epsilon H H^{T}+\frac{1}{\epsilon} M^{T} M
$$

Suppose now that a process described by (35) for which (36) and (37) hold is asymptotically stable. Then extending the previous control law design analysis to this case is not straightforward since, in particular, the limit profile stability conditions depend in a very complex way on the matrices defining the uncertainty. Further analysis, however, leads to the following result which allows control law design.

Theorem 4 Suppose that a control law of the form (6) is applied to a discrete linear repetitive process described by (35) with uncertainty satisfying (36) and (37). Then the resulting controlled process is strongly practically stable if the following LMIs hold

$$
\begin{aligned}
& {\left[\begin{array}{ccc}
W_{2}-G_{2}-G_{2}^{T} & * & * \\
D_{0} G_{2}+D R_{2} & -W_{2}+\epsilon_{1} H_{2} H_{2}^{T} & * \\
M_{2} G_{2}+M_{3} R_{2} & 0 & -\epsilon_{1} I
\end{array}\right]<0} \\
& {\left[\begin{array}{ccc}
W_{1}-G_{1}-G_{1}^{T} & * & * \\
A G_{1}+B R_{1} & -W_{1}+\epsilon_{2} H_{1} H_{1}^{T} & * \\
M_{1} G_{1}+M_{3} R_{1} & 0 & -\epsilon_{2} I
\end{array}\right]<0} \\
& {\left[\begin{array}{c}
-X_{11}^{1}+A G_{1}+B R_{1}+\left(A G_{1}+B R_{1}\right)^{T} \\
+4 \epsilon_{3} H_{1} H_{1}^{T} \\
C G_{1}+D R_{1}+\left(B_{0} G_{2}+B R_{2}\right)^{T}+4 \epsilon_{3} H_{2} H_{1}^{T}
\end{array}\right.} \\
& -G_{1}+\left(A G_{1} \beta_{1}+B R_{1} \beta_{1}\right)^{T} \\
& \left(B_{0} G_{2} \beta_{1}+B R_{2} \beta_{1}\right)^{T} \\
& M_{1} G_{1}+M_{3} R_{1} \\
& 0 \\
& 0 \\
& 0 \\
& D_{0} G_{2}-G_{2}+D R_{2}+{ }^{*}\left(D_{0} G_{2}-G_{2}+D R_{2}\right)^{T} \\
& +4 \epsilon_{3} H_{2} H_{2}^{T} \\
& \left(C G_{1} \beta_{1}+D R_{1} \beta_{1}\right)^{T} \\
& Y_{22}^{1}-G_{2}+\left(D_{0} G_{2} \beta_{1}-G_{2} \beta_{1}+D R_{2} \beta_{1}\right)^{T} \\
& 0 \\
& M_{2} G_{2}+M_{3} R_{2} \\
& 0 \\
& 0 \\
& X_{21}^{1}
\end{aligned}
$$




$$
\begin{aligned}
& \left.\begin{array}{ccccc}
* & * & * & * & * \\
* & * & * & * & * \\
* & * & * & * & * \\
X_{22}^{1}-G_{2} \beta_{1}-\left(G_{2} \beta_{1}\right)^{T} & * & * & * & * \\
0 & -\epsilon_{3} I & * & * & * \\
0 & 0 & -\epsilon_{3} I & * & * \\
0 & 0 & 0 & -\epsilon_{3} I & * \\
M_{2} G_{2} \beta_{1}+M_{3} R_{2} \beta_{1} & 0 & 0 & 0 & -\epsilon_{3} I
\end{array}\right]<0 \\
& {\left[\begin{array}{c}
A G_{1}-G_{1}+B R_{1}+\left(A G_{1}-G_{1}+B R_{1}\right)^{T} \\
+4 \epsilon_{4} H_{1} H_{1}^{T} \\
C G_{1}+D R_{1}+\left(B_{0} G_{2}+B R_{2}\right)^{T}+4 \epsilon_{4} H_{2} H_{1}^{T}
\end{array}\right.} \\
& Y_{11}^{2}-G_{1}+\left(A G_{1} \beta_{2}-G_{1} \beta_{2}+B R_{1} \beta_{2}\right)^{T} \\
& \left(B_{0} G_{2} \beta_{2}+B R_{2} \beta_{2}\right)^{T} \\
& M_{1} G_{1}+M_{3} R_{1} \\
& 0 \\
& 0 \\
& 0 \\
& -X_{22}^{2}+\stackrel{*}{D}_{0} G_{2}+D R_{2}+\left(D_{0} G_{2}+D R_{2}\right)^{T} \\
& +4 \epsilon_{4} H_{2} H_{2}^{T} \\
& \left(C G_{1} \beta_{2}+D R_{1} \beta_{2}\right)^{T} \\
& -G_{2}+\left(D_{0} G_{2} \beta_{2}+D R_{2} \beta_{2}\right)^{T} \\
& 0 \\
& M_{2} G_{2}+M_{3} R_{2} \\
& 0 \\
& 0 \\
& X_{11}^{2}-G_{1} \beta_{2}-\left(G_{1} \beta_{2}\right)^{T} \\
& X_{21}^{2} \\
& 0 \\
& 0 \\
& M_{1} G_{1} \beta_{1}+M_{3} R_{1} \beta_{1} \\
& 0 \\
& \left.\begin{array}{ccccc}
* & * & * & * & * \\
* & * & * & * & * \\
* & * & * & * & * \\
X_{22}^{2}-G_{2} \beta_{2}-\left(G_{2} \beta_{2}\right)^{T} & * & * & * & * \\
0 & -\epsilon_{4} I & * & * & * \\
0 & 0 & -\epsilon_{4} I & * & * \\
0 & 0 & 0 & -\epsilon_{4} I & * \\
M_{2} G_{2} \beta_{1}+M_{3} R_{2} \beta_{1} & 0 & 0 & 0 & -\epsilon_{4} I
\end{array}\right]<0
\end{aligned}
$$

for a given $\beta_{1}>1, \beta_{2}>1$ with the variables

$$
\begin{aligned}
& W_{1}>0, W_{2}>0, X_{11}^{1}=\left(X_{11}^{1}\right)^{T}, X_{22}^{1}=\left(X_{22}^{1}\right)^{T}, X_{11}^{2}=\left(X_{11}^{2}\right)^{T}, X_{22}^{2}=\left(X_{22}^{2}\right)^{T}, X_{21}^{1}, \\
& X_{21}^{2}, Y_{11}^{2}, Y_{22}^{1}, G_{1}, G_{2}, R_{1}, R_{2} \epsilon_{1}>0 \epsilon_{2}>0 \epsilon_{3}>0 \epsilon_{4}>0 .
\end{aligned}
$$

When these conditions hold, stabilizing control law matrices are given by

$$
K_{1}=R_{1} G_{1}^{-1}, \quad K_{2}=R_{2} G_{2}^{-1}
$$

Proof The proof relies on representing the conditions for strong practical stability of the controlled process in LMI form. This can be done by the use of the results of Lemmas 4 and 5 followed by appropriate application of the Schur's complement formula and congruence transforms. 
Here the nominal model is again (1) but now the uncertainty is defined by the matrix polytope

$$
\left[\begin{array}{lll}
A & B_{0} & B \\
C & D_{0} & D
\end{array}\right] \in C o\left(\left[\begin{array}{ccc}
A^{i} & B_{0}^{i} & B^{i} \\
C^{i} & D_{0}^{i} & D^{i}
\end{array}\right]\right), \quad i=1,2, \ldots, h
$$

and

$$
C o\left(\left[\begin{array}{ccc}
A^{i} & B_{0}^{i} & B^{i} \\
C^{i} & D_{0}^{i} & D^{i}
\end{array}\right]\right):=\left\{X:=\sum_{i=1}^{h} \alpha_{i}\left[\begin{array}{lll}
A^{i} & B_{0}^{i} & B^{i} \\
C^{i} & D_{0}^{i} & D^{i}
\end{array}\right], \alpha_{i} \geq 0, \sum_{i=1}^{h} \alpha_{i}=1\right\}
$$

We also require the following result (Chaabane et al. 2007).

Lemma 6 Consider a $1 D$ discrete singular linear system described by (7) where the statespace matrices are subject to uncertainty of the form defined by (44) and (45) (as applied to this $1 D$ case) under the action of a control law of the form (27). Then the resulting system is admissible if there exist matrices $X_{i}, Y_{i}$, for $i=1,2, \ldots, h$, and two matrices $G$ and $R$ such that the LMI

$$
\left[\begin{array}{cc}
-E X_{i} E^{T} & \left(E^{\perp} Y_{i} E^{\ddagger}\right)^{T} \\
E^{\perp} Y_{i} E^{\ddagger} & X_{i}
\end{array}\right]+S y m\left\{\left[\begin{array}{c}
A^{i} G+B^{i} R \\
-G
\end{array}\right]\left[\begin{array}{ll}
U^{-T} & \beta V^{T}
\end{array}\right]\right\}<0
$$

is feasible, for a given $\beta>1$. Also

$$
K=R G^{-1}
$$

results in a stable system.

Now we have the main result for the case of polytopic uncertainty.

Theorem 5 Suppose that a control law of the form (6) is applied to a discrete linear repetitive process described by (35) with uncertainty modeled by (44) and (45). Then the resulting controlled process is strongly practically stable if, the following LMIs hold

$$
\begin{aligned}
& {\left[\begin{array}{cc}
W_{2}-G_{2}-G_{2}^{T} & * \\
D_{0}^{i} G_{2}+D^{i} R_{2} & -W_{2}
\end{array}\right]<0 } \\
& {\left[\begin{array}{cc}
W_{1}-G_{1}-G_{1}^{T} & * \\
A^{i} G_{1}+B^{i} R_{1} & -W_{1}
\end{array}\right]<0 } \\
& {\left[\begin{array}{cccc}
-X_{11}^{1 i} & 0 & 0 & 0 \\
0 & 0 & 0 & \left(Y_{22}^{1 i}\right)^{T} \\
0 & 0 & X_{11}^{1 i}\left(X_{21}^{1 i}\right)^{T} \\
0 & Y_{22}^{1 i} & X_{21}^{1 i} & X_{22}^{1 i}
\end{array}\right]+\operatorname{Sym}\left\{\begin{array}{cc}
A^{i} G_{1}+B^{i} R_{1} & B_{0}^{i} G_{2}+B^{i} R_{2} \\
C^{i} G_{1}+D^{i} R_{1} & D_{0}^{i} G_{2}-G_{2}+D^{i} R_{2} \\
-G_{1} & 0 \\
0 & -G_{2}
\end{array}\right.}
\end{aligned}
$$




$$
\begin{aligned}
& \left.\begin{array}{cc}
A^{i} G_{1} \beta_{1}+B^{i} R_{1} \beta_{1} & B_{0}^{i} G_{2} \beta_{1}+B^{i} R_{2} \beta_{1} \\
C^{i} G_{1} \beta_{1}+D^{i} R_{1} \beta_{1} & D_{0}^{i} G_{2} \beta_{1}-G_{2} \beta_{1}+D^{i} R_{2} \beta_{1} \\
-G_{1} \beta_{1} & 0 \\
0 & -G_{2} \beta_{1}
\end{array}\right\}<0 \\
& {\left[\begin{array}{cccc}
0 & 0 & \left(Y_{11}^{2 i}\right)^{T} & 0 \\
0 & -X_{22}^{2 i} & 0 & 0 \\
Y_{11}^{2 i} & 0 & X_{11}^{2 i} & \left(X_{21}^{2 i}\right)^{T} \\
0 & 0 & X_{21}^{2 i} & X_{22}^{2 i}
\end{array}\right]+\operatorname{Sym}\left\{\begin{array}{cc}
A^{i} G_{1}-G_{1}+B^{i} R_{1} & B_{0}^{i} G_{2}+B^{i} R_{2} \\
C^{i} G_{1}+D^{i} R_{1} & D_{0}^{i} G_{2}+D^{i} R_{2} \\
-G_{1} & 0 \\
0 & -G_{2}
\end{array}\right.} \\
& \left.\begin{array}{cc}
A^{i} G_{1} \beta_{2}-G_{1} \beta_{2}+B^{i} R_{1} \beta_{2} & B_{0}^{i} G_{2} \beta_{2}+B^{i} R_{2} \beta_{2} \\
C^{i} G_{1} \beta_{2}+D^{i} R_{1} \beta_{2} & D_{0}^{i} G_{2} \beta_{2}+D^{i} R_{2} \beta_{2} \\
-G_{1} \beta_{2} & 0 \\
0 & -G_{2} \beta_{2}
\end{array}\right\}<0
\end{aligned}
$$

for given $\beta_{1}>1$ and $\beta_{2}>1$ with the variables

$$
\begin{aligned}
& W_{1}>0, W_{2}>0, X_{21}^{1 i}, X_{21}^{2 i}, X_{11}^{1 i}=\left(X_{11}^{1 i}\right)^{T}, X_{22}^{1 i}=\left(X_{22}^{1 i}\right)^{T}, Y_{11}^{2 i}, Y_{22}^{1 i}, X_{11}^{2 i}=\left(X_{11}^{2 i}\right)^{T}, \\
& X_{22}^{2 i}=\left(X_{22}^{2 i}\right)^{T}, G_{1}, G_{2}, R_{1}, R_{2}
\end{aligned}
$$

When these conditions hold, stabilizing control law matrices are given by

$$
\begin{aligned}
& K_{1}=R_{1} G_{1}^{-1} \\
& K_{2}=R_{2} G_{2}^{-1}
\end{aligned}
$$

Proof The proof relies on representing the conditions for strong practical stability in LMI form. This can be done by use of Lemma 6, followed by appropriate application of the Schur's complement formula and congruence transforms. Hence the details are omitted here.

\section{An illustrative example}

As an example consider a simplified model of a metal rolling process, see Bochniak et al. (2008) for the details, where $\alpha=100$ and

$$
\left[\begin{array}{l|l|l}
A & B_{0} & B \\
\hline C & D_{0} & D
\end{array}\right]=10^{-3} \times\left[\begin{array}{rr|r|l}
972.0 & 97.2 & 7.94 & -0.278 \\
-278.0 & 972.0 & 79.4 & -2.78 \\
\hline 972.0 & 97.2 & 722.0 & -0.278
\end{array}\right]
$$

with boundary conditions

$$
\begin{aligned}
x_{k+1}(0) & =\left[\begin{array}{ll}
-10, & -10
\end{array}\right]^{T}, \quad k \geq 0 \\
y_{0}(p) & =-20, \quad 0 \leq p \leq \alpha-1
\end{aligned}
$$

This process is practically stable (i.e. $r\left(D_{0}<1\right.$ and $\left.r(A)<1\right)$ and Fig. 5 shows pass profile sequence generated (with zero control input). This is not acceptable in metal rolling due to the oscillations present in the along the pass dynamics. One option here would be to attempt control law design to ensure practical stability without these oscillations but, as noted above, there will be cases when this property is simply not strong enough. Here we give representative results from control law design for strong practical stability where we also have the design variables $\beta_{1}$ and $\beta_{2}$ available to assist in obtaining acceptable along the pass dynamics. 


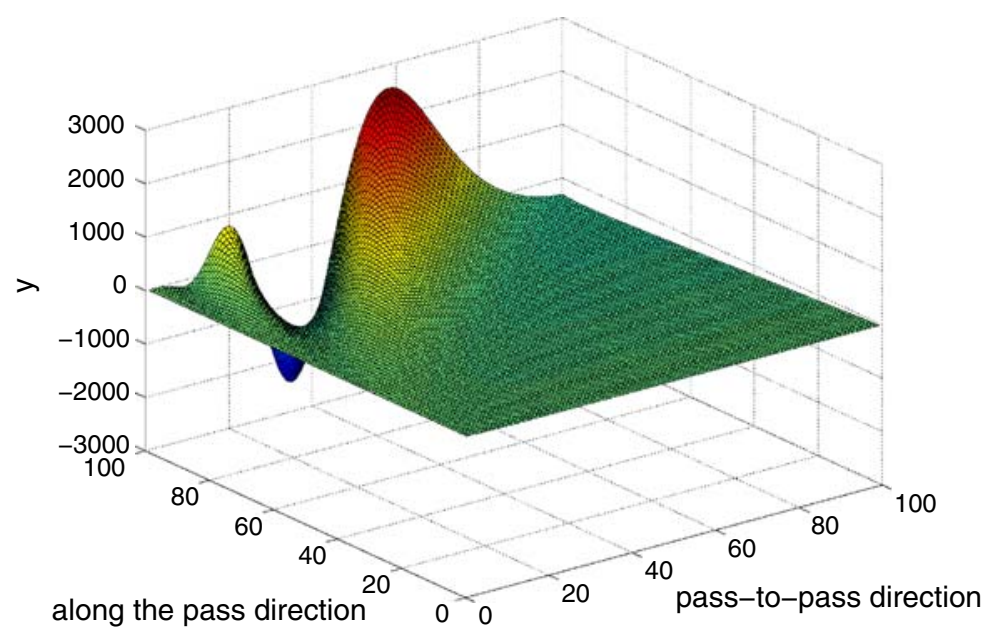

Fig. 5 Pass profile dynamics — practically stable

Solving the LMIs of Theorem 3 gives the control law matrices

(a) for $\beta_{1}=19.0, \quad \beta_{2}=15.0$

$$
K_{1}=\left[\begin{array}{ll}
1600.0352 .0
\end{array}\right] \quad K_{2}=35.3
$$

(b) for $\beta_{1}=429.0, \quad \beta_{2}=515.0$

$$
K_{1}=\left[\begin{array}{ll}
3320.0352 .0
\end{array}\right] \quad K_{2}=28.9
$$

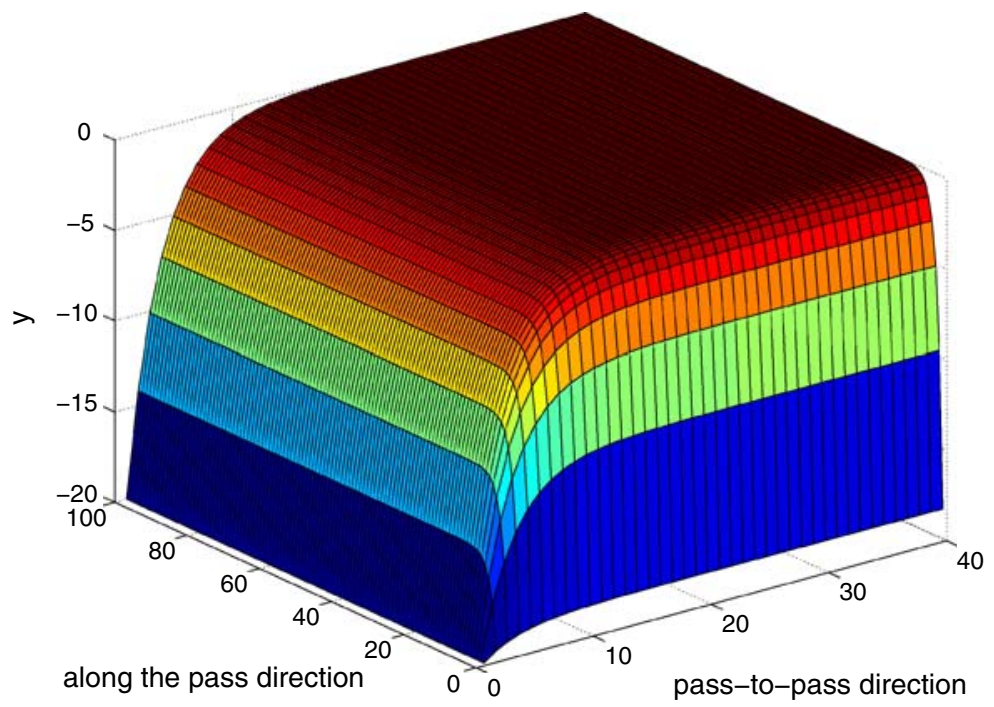

Fig. 6 Pass profile dynamics - controlled process with $\beta_{1}=19.0, \beta_{2}=15.0$ 


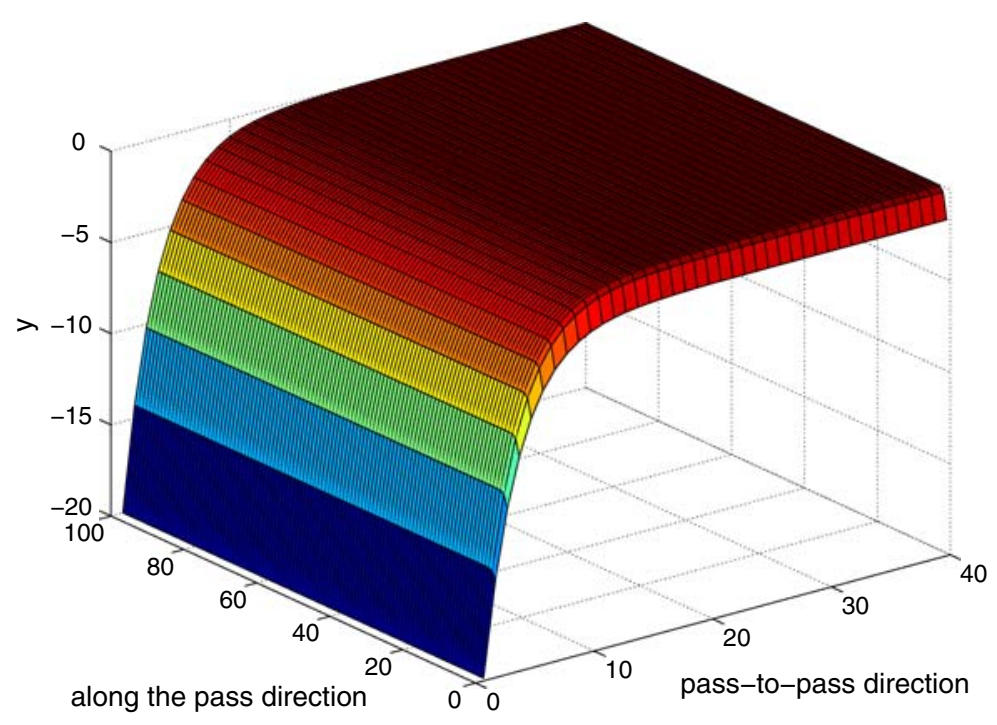

Fig. 7 Pass profile dynamics - controlled process with $\beta_{1}=429.0$ and $\beta_{2}=515.0$

Sequences of pass profiles dynamics generated by the controlled process are given in Figs. 6 and 7 respectively.

Examining these simulations we see that the sequence of pass profiles is converging in the pass-to-pas direction, will eventually reach the limit profile described by a stable 1D discrete linear system, and hence the possibility that a limit profile with acceptable (along the pass) transient response can be achieved. Moreover, unacceptable oscillations do not appear in the along the pass dynamics.

\section{Conclusions}

In this paper a new concept of stability for discrete linear repetitive processes has been developed from physical motivations and it has been shown that it can be expressed in terms of conditions that can be tested for numerical examples by LMIs. It has also been shown how these tests lead naturally to control law design to ensure this property, including the case when there is uncertainty associated with the process state-space model. The resulting design algorithms also include parameters which can be used to tune performance-a highly desirable feature in terms of applications in both signal processing/filtering and control. Further work includes the development of methods to tune these parameters to obtained stability plus desired performance.

Acknowledgements This work has been partially supported by the Polish Ministry of Science and Higher Education under the project N N514 293235.

\section{References}

Agathoklis, P. \& Bruton, L. T. (1983). Practical-BIBO stability of n dimensional discrete systems. Proceedings of The Institution of Electrical Engineers, 130, 236-242.

Amann, N., Owens, D. H., \& Rogers, E. (1998). Predictive optimal iterative learning control. International Journal of Control, 69(2), 203-226. 
Bochniak, J., Galkowski, K., \& Rogers, E. (2008). Multi-machine operations modelled and controlled as switched linear repetitive processes. International Journal of Control, 81(10), 1549-1567.

Boland, F. M., \& Owens, D. H. (1980). Linear multipass processes-a two-dimensional systems interpretation. Proceedings of the Institution of Electrical Engineers, 127, 189-193.

Chaabane, M., Bachelier, O., \& Mehdi, D. (2007). Admissibility and state feedback stabilization of discrete singular systems: An LMI Approach, LAII-ESIP Technical Report 20070208DM, Poitiers, France.

Dai, L. (1989). Singular control systems. Springer.

de Oliveira, M. C., Bernussou, J., \& Geromel, J. C. (1999). A new discrete-time robust stability condition. Systems and Control Letters, 37(4), 261-265.

Fornasini, E., \& Marchesini, G. (1978). Doubly indexed dynamical systems: State-space models and structural properities. Mathematical System Theory, 12, 59-72.

Galkowski, K., Rogers, E., Xu, S., Lam, J., \& Owens, D. H. (2002). LMIs-a fundamental tool in analysis and controller design for discrete linear repetitive processes. IEEE Transactions on Circuits and Systems I: Fundamental Theory and Applications, 49(6), 768-778.

Peaucelle, D., Arzelier, D., Bachelier, O., \& Bernussou, J. (2000). A new robust D-stability condition for real convex polytopic uncertainty. Systems and Control Letters, 40(1), 21-30.

Ratcliffe, J. D., Lewin, P. L., Rogers, E., Hatonen, J. J., \& Owens, D. H. (2006). Norm-optimal iterative learning control applied to gantry robots for automation applications. IEEE Transactions on Robotics, 22(6), 1303-1307.

Roberts, P. D. (2002). Two-dimensional analysis of an iterative nonlinear optimal control algorithm. IEEE Transactions on Circuits and Systems I: Fundamental Theory and Applications, 49(6), 872-878.

Roesser, R. P. (1975). A discrete state-space model for linear image processing. IEEE Transactions on Automatic Control, AC-20, 1-10.

Rogers, E., Galkowski, K., \& Owens, D. H. (2007). Control systems theory and applications for linear repetitive processes. Lecture Notes in Control and Information Sciences Series (Vol. 349). Springer.

Wood, J., Oberst, U., Rogers, E., \& Owens, H. H. (2000). A behavioural approach to the pole structure of one-dimensional and multidimensional linear systems. SIAM Journal on Control and Optimization, 38(2) $627-661$.

Wu, L., Lam, J., Paszke, W., Galkowski, K., \& Rogers, E. (2009). Control and filtering for discrete linear repetitive processes with $\mathcal{H}_{\infty}$ and $l_{2}-l_{\infty}$ performance. International Journal of Multidimensional Systems and Signal Processing (in print).

Xu, L., Saito, O., \& Abe, K. (1994). The design of practically stable nD feedback systems. Automatica, 30(9), 1389-1397.

Xu, L., Saito, O., \& Abe, K., (1997). nD control systems in a practical sense. International Journal of Applied Mathematics and Computer Science, 7(4), 907-941.

\section{Author Biographies}

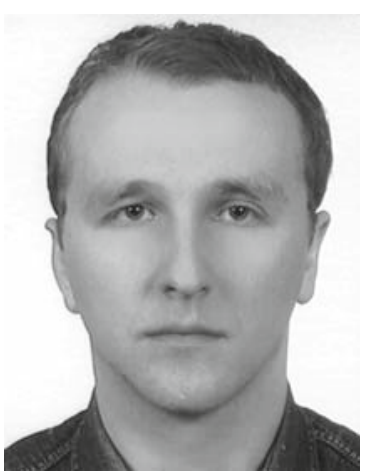

Paweł Dabkowski was born in Torun in 1982. He received the M.Sc degree at the Nicolaus Copernicus University of Torun, Poland in 2006. Currently he is a Ph.D student at the Institute of Physics, Nicolaus Copernicus University in Torun. His research interests include the control theory and multidimensional systems, especially the linear repetitive processes. 


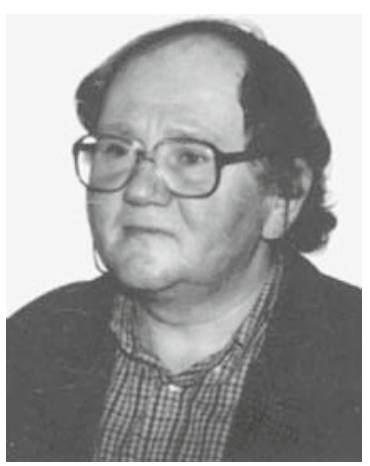

Krzysztof Gałkowski received the M.S., Ph.D. and Habilitation (D.Sc.) degrees in Electronics/Automatic Control from Technical University of Wrocław, Poland in 1972, 1977 and 1994 respectively. In October 1996 he joined the Technical University of Zielona Góra (now the University of Zielona Góra), Poland where he holds the professor position, and he is a visiting professor in the School of Electronics and Computer Science, University of Southampton, UK. In 2002, he was awarded the degree "Professor of Technical Science" the highest scientific degree in Poland. He spent academic year 2004-2005 and 2006-2007 in The University of Wuppertal, Germany as and awardee of The Gerhard Mercator Guest Professor funded by DFG. He holds also the Professor position at The Nicolaus Copernicus University in Torun, Poland at The Department of Physics, Astarnomy and Computer Science. He is an Associate Editor of International Journal of Multidimensional Systems and Signal Processing, International Journal of Control and International Journal of Applied Mathematics and Computer Science. He has co-organised four International Workshops NDS 1998, Lagov, Poland, NDS 2000, Czocha Castle, Poland, the third as a Symposium within MTNS 2002 in Notre Dame IN, US, and the last one NDS 2005 in Wuppertal, Germany. In 2007 NDS 2007 has been organized in Aveiro, Portugal and in 2009 will be at Thessaloniki. In 2004, he obtained a Siemens Award for his research contributions. His research interests include multidimensional (nD) systems and repetitive processes-theory and applications, control and related numerical and symbolic algebra methods.

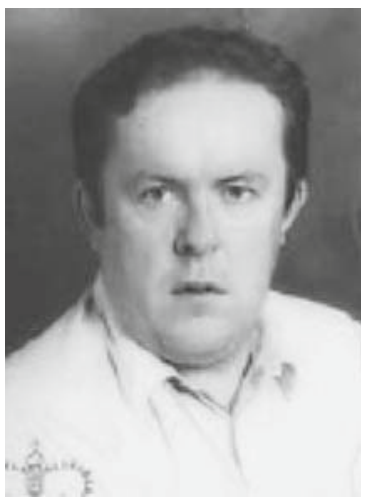

Eric Rogers was born in 1956 near Dungannon in Northern Ireland. He read Mechanical Engineering as an undergraduate in Queen's University, Belfast, UK and was awarded his Ph.D. degree by The University of Sheffield, UK for a thesis in the area of multidimensional systems theory. Recently he has been awarded the D.Sc. degree by Queen's University Belfast for research in $\mathrm{nD}$ systems theory and applications. He has been with The University of Southampton, UK since 1990 where he is currently Professor of Control Systems Theory and Design in The School of Electronics and Computer Science. His current major research interests include multidimensional systems theory and applications, with particular emphasis on behavioral systems theory approaches and systems with repetitive dynamics, iterative learning control, flow control, and active control of microvibrations. He is currently the Editor of The International Journal of Control, an Associate Editor of Multidimensional Systems and Signal Processing, and a member of the editorial board of Applied Mathematics and Computer Science. In addition, he has served extensively on IEEE, IFAC and IEE technical committees and acted as a consultant to numerous companies and government agencies in the UK and abroad.

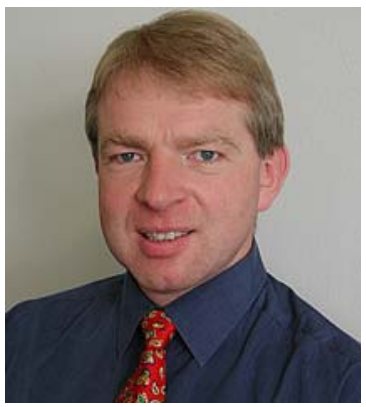

Anton Kummert was born in Amberg, Germany on April 6, 1959. He received the Dipl.-Ing. (FH) degree in Electrical Engineering from Fachhochschule Coburg, Coburg, Germany, in 1982, and the Dipl.-Ing. and Dr.-Ing. degrees from Ruhr-UniversitSt Bochum, Bochum, Germany, in 1985 and 1988, respectively. From 1985 to 1991, he was a Research Assistant at the Department of Electrical Engineering of Ruhr-UniversitSt Bochum. From 1991 to 1995, he was employed by STN Atlas Elektronik, Bremen, Germany. Since 1995, he has been a Professor for Communication Theory at University of Wuppertal, Wuppertal, Germany. Mr. Kummert is a recipient of the "Heinrich-Kost-Preis" (1989) and of the "Akademie-Preis 1990" of Rheinisch-WestfSlischeAkademie der Wissenschaften (1990). He is a member of Informationstechnische Gesellschaft, Germany (ITG) and Senior member of IEEE. He is and has been member of the editorial board of several international journals, organizer and chairman of numerous sessions at major conferences, and one of the General Chairs and organizers of the 4th International Workshop on Multidimensional (ND) Systems. His current research interests comprise multidimensional systems and circuit theory, image processing and generation, wireless ad hoc networks, and tomographic reconstruction methods. 